\title{
Application of Wavelet Analysis in the Vibration Signal of Diesel Engine Noise Reduction Techniques
}

\author{
Gen Fan ${ }^{1, a}$, Wenbin Liu $^{1, b}$ \\ ${ }^{1}$ Wuhan Technology and Business University , Wuhan 430065, China \\ a709232896@qq.com; b812926096@qq.com
}

Keywords: Wavelet Analysis, Denoising, Threshold

\begin{abstract}
This paper synthetically analyzes and compares classical denoising methods, discussing their respective field of application, their denoising performance and influencing factors, etc. We summarize the advantages and disadvantages of these methods. Finally, vibration acceleration signal sampled on Diesel engine was de-noised by wavelet analysis and wavelet packet analysis, and its SNR(signal-noise-ratio) is enhanced to a satisfactory degree.
\end{abstract}

\section{Introduction}

Wavelet transform is a new signal analysis method, has been widely studied and applied, for signal processing methods continue to emerge out of the use of wavelet, this paper discusses the basic theory of wavelet transform, the characteristics of wavelet transform are summarized and analyzed, and the decomposition denoising wavelet analysis and wavelet packet analysis theory is applied to diesel engine engine surface vibration signal.

\section{Wavelet Denoising Algorithm, Review}

The current detection signal of one-dimensional wavelet denoising method, can be roughly divided into the decomposition and reconstruction method, the nonlinear wavelet threshold denoising method, the translation invariant method and the wavelet transform modulus maxima method. The following are some detailed analysis and comparison of four kinds of denoising method.

The decomposition and reconstruction method in specific circumstances, for the deterministic noise, the prior knowledge can determine the frequency range in advance, especially effective in signal and noise bands do not overlap each other when. The advantage of this method is that the algorithm is simple, the calculation speed is fast; the disadvantage is not widely applicable scope. As a practical application in signal mixed with white noise, the denoising effect is poor.

Wavelet threshold denoising method is the simplest to implement, a method to calculate the minimum amount, and thus made the most widely used. But the threshold value selection is difficult, although Donoho is proved in theory and to find the optimal universal threshold, but not the actual application result is very satisfactory. In addition, the variance threshold selection is also dependent on the noise, therefore require prior noise variance estimation. Threshold selection directly affects the results of denoising. In addition, it is also important to select the threshold function, usually the hard threshold function and the soft threshold function because of its own shortcomings, de-noising effect is not ideal.

Translation invariant denoising method is essentially improvement on threshold method, mainly is suitable for the signal mixed with white noise and contain some discontinuous points, but to the circular shift on the signal, so bring a large amount of calculation, the calculation speed is slow. The utility model has the advantages of effectively removing the Pseudo-Gibbs phenomenon and the elimination of saw tooth phenomenon, can approximate the original signal better.

Modulus maxima denoising method principle is different according to the change characteristics of signal and noise under wavelet transform with scale changes is shown and raised, have very good guarantee theory, so the denoising performance is very stable, it is less dependent on the noise, 
there is no need to know the noise variance, and the low the signal-to-noise ratio of the signal denoising superiority. However, it has a fundamental drawback is the existence of a by the modulus maxima of wavelet coefficients of the reconstruction problem in the denoising process, so as to make the calculation amount greatly increased the method, because often can not meet the actual processing system for real-time property of the algorithm requires to lose its application value. In addition, the denoising effect is not very satisfactory.

Because all kinds of denoising methods exist parameter selection problem, different parameters corresponding out denoising results will have some differences, therefore, is not good in general will be a numerical comparison of the four methods.

\section{Based on Wavelet Analysis of Diesel Surface Vibration Signals Noise Reduction Machine Technology}

The diesel engine is a mechanical system is a very complex, vibration excitation source, the pressure in the cylinder, the piston reciprocating inertia force, side thrust, crankshaft unbalance force, impact, into the exhaust gas threshold impact, the impact of main bearing of main shaft neck and so on are caused by diesel engine vibration. Thus the measured vibrational information to include a variety of ingredients and interference, which belongs to the non stationary vibration signal. Only effectively eliminate the interference and noise signal, obtain the useful signal, it is possible to improve the diagnosis accuracy, get the conclusion of analysis and reliable.

The traditional noise reduction methods of signal processing is the low pass filtering method based on $\mathrm{Fu}$ Liye analysis, the signal from the frequency domain filtering the high frequency part and retained in the low frequency part. Generally the useful signal concentrates on the low frequency part, the noise distribution in the high-frequency part, can use the low pass filtering method for noise reduction. Filtering method based on Fu Liye analysis for non-stationary signal is suitable, but for the non-stationary signal, it can be useful to the high frequency part of signal and the high frequency interference caused by noise due to the distinction between. If the cutoff frequency of the lowpass filter is too high, there is still a lot of noise in the filtered signal, on the other hand, then some useful signals will be filtered out, this will result in the loss of useful information.

1.Wavelet analysis of signal characteristics

Frequency localization function with adaptive time - orthogonal wavelet decomposition, mutations in some parts of the signal, wavelet components performance greatly, it and noise in the high frequency part of the uniform performance just formed obvious contrast, so orthogonal wavelet decomposition can effectively distinguish the mutation part and the noise in the signal. Similarly, the orthogonal wavelet packet decomposition can not only more meticulous performance signal mutation high frequency parts, also can obvious noise high-frequency low-frequency signal in the hybrid. Therefore, wavelet decomposition and wavelet packet decomposition can effectively denoising non-stationary signal.

Using noisy square wave signal (Figure 1) is simulated, using the DB2 wavelet orthogonal wavelet decomposition, the signal in different scale details wavelet transform values as in figure 2 . We can see that, with increasing of scale, in the detail parts of the signal, with the increasing of scale wavelet transform, signal mutation at the value increases obviously, and the noise by wavelet transform is invariable, the useful signal and noise is effectively separated out. In the detail parts of the signal, using an appropriate threshold, the absolute value of the wavelet transform is less than a threshold value of zero, it can keep the useful in high frequency signal at the same time, eliminate most noise signal. Principle of wavelet packet denoising principle and wavelet denoising is the same. 


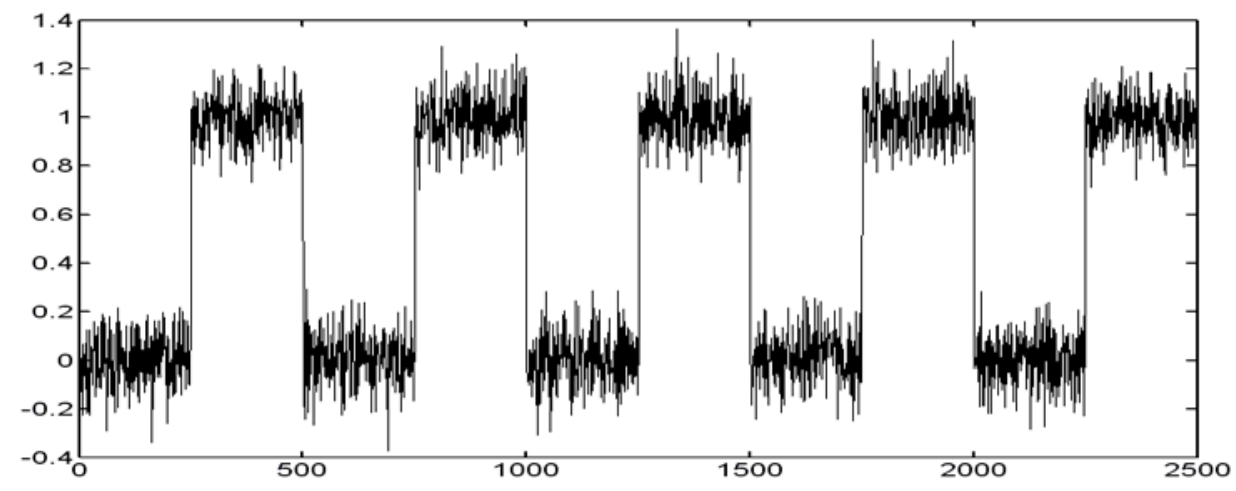

Figure 1 noisy square wave signal

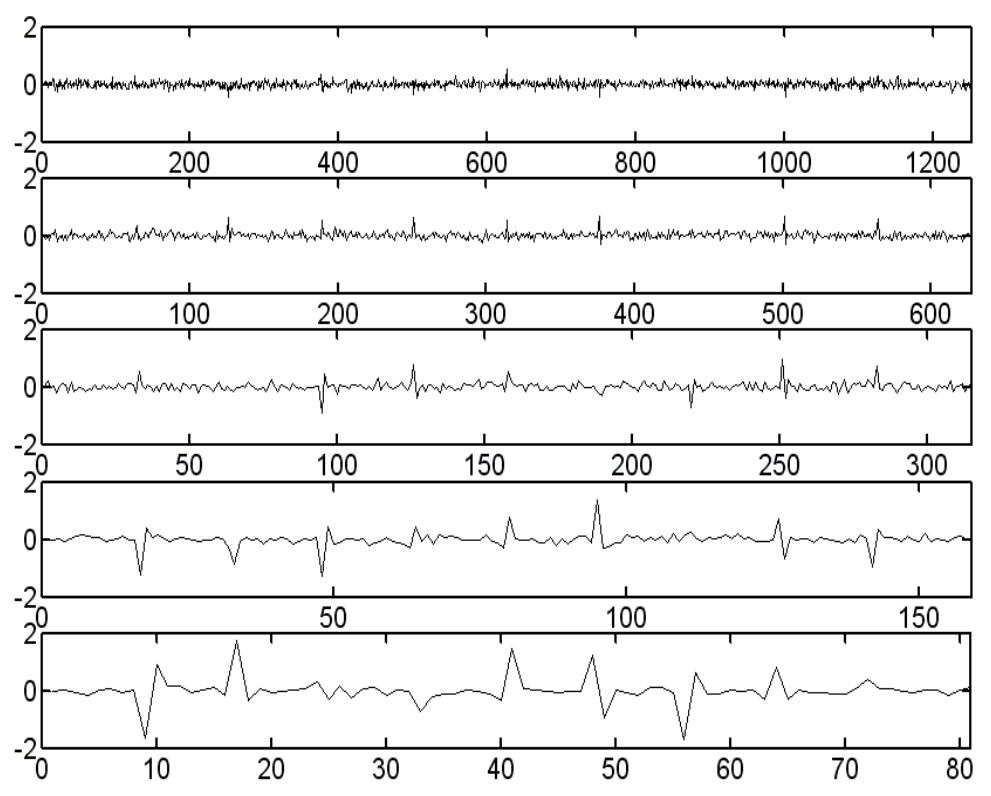

Figure 2 noisy square wave signal decomposition results (details)

2.The process of wavelet threshold denoising

In the actual project, the useful signal is usually low frequency signal or are some of the more stable signals, and the noise signal is shown as the high frequency signal is usually. Signal denoising process can be divided into three steps:

(1) wavelet decomposition of signal. The choice of a wavelet, and determine the number of layers of wavelet decomposition, and then the signal is Ceng Xiaobo decomposition;

(2) threshold quantization high-frequency coefficients of wavelet decomposition. The high frequency coefficients of each layer of the first layer to layer, select the appropriate threshold and threshold quantization.

(3) reconstruction of one dimensional wavelet. Based on the wavelet decomposition of the second layer of the low frequency coefficients and the quantized treatment after the first layer to the second layer of the high frequency coefficients of wavelet, signal reconstruction.

In the three step, the key is how to select the threshold and how to quantify the quality threshold, it is directly related to the signal denoising. 


\section{Based on Wavelet Packet Denoising Algorithm}

The main advantages of the wavelet package relative Yu Xiaobo is the high frequency part of signal wavelet packet can do a more detailed description, the analysis of the signal ability is stronger, therefore put forward using wavelet packet decomposition of signal denoising. By using wavelet packet denoising procedure is as follows:

1.Decomposition

Wavelet for signal using the given layer wavelet packet decomposition.

2.Calculation of the optimal (optimal) tree

The best subtree calculating initial tree according to the given entropy standard. Through the Matlab can quickly and easily calculate the best subtree.

3.The wavelet packet coefficients by threshold processing

For each wavelet packet coefficient (except the low frequency part), the choice of a threshold to process the. Such as when using the SURE (Stein 's Unbiased Estimate of Risk) entropy criteria to select threshold denoising, signal length is set, then the threshold for the $s=\sqrt{2^{*} \ln \left(l^{*} \log _{2} l\right)}$

4.reconstruction

In the initial signal the $\mathrm{N}$ layer low frequency coefficients and the high frequency coefficients by threshold after treatment based on wavelet packet reconstruction.

For example, on a signal by wavelet packet denoising, the signal for Matlab 6.5 inside a sample signal (noischir.mat) as shown in Figure 4.1, the original signal length is 1024, then the calculated by using SURE entropy criterion when the threshold is 4.2975 . Wavelet packet basis function using wavelet DB2, decomposition level is 3, the wavelet packet decomposition as shown in Figure 3.

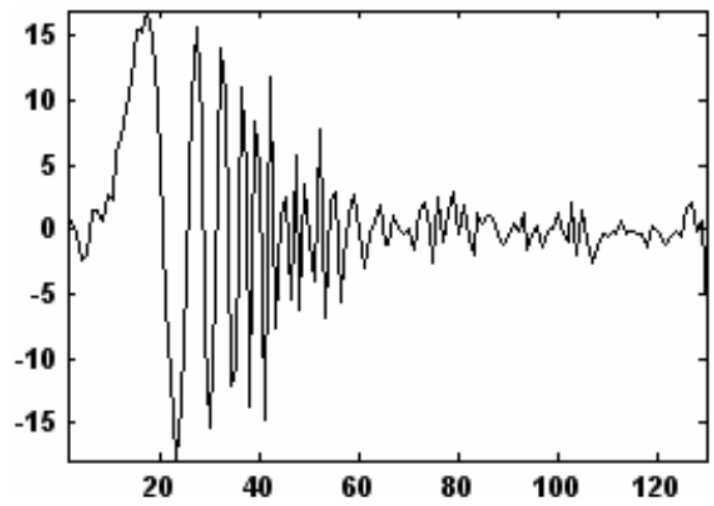

(1)

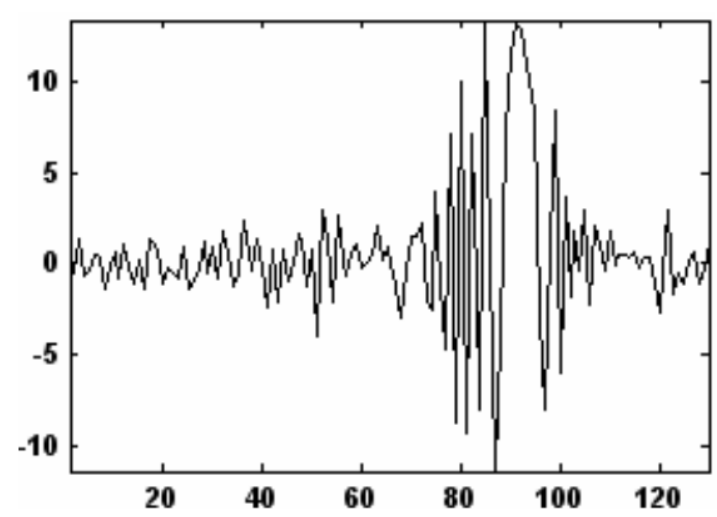

(3)

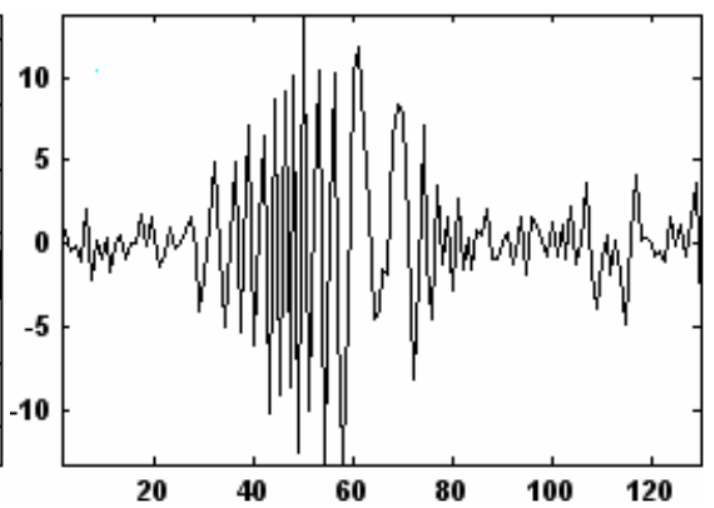

(2)

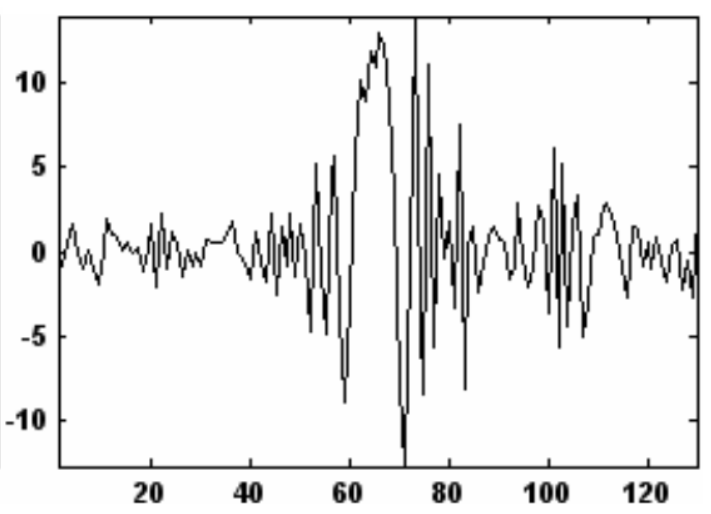

(4)

Fig. 3 the wavelet packet decomposition

4.Application of wavelet packet denoising to diesel engine surface vibration signal Wavelet packet is used for noise reduction of diesel engine surface vibration signal, the processing procedure is as follows: 
(1) will be layer wavelet packet decomposition of the signal with noise;

(2) the best subtree to calculate the initial tree according to the given entropy standard;

(3) for each wavelet packet coefficient (except the low frequency part), the choice of a threshold to process the. If the selected threshold is not appropriate, it may be the threshold quantization process, until a satisfactory threshold.

(4) the initial signal layer low frequency coefficients and high frequency coefficients after after processing by wavelet reconstruction.

This procedure makes the noise elimination, and be better retain edge information.

This paper uses DB2 wavelet, on the cylinder head vibration signals of the four layer wavelet packet decomposition, threshold denoising. Because of unknown properties of diesel engine noise signal, so using heuristic threshold for signal processing. Figure 5 is the use of threshold denoising based on wavelet packet method were compared before and after noise waveform, noise reduction remained after the signal useful spikes and mutation part, and eliminates the noise of diesel engine.

\section{The Summary of This Chapter}

This paper mainly introduces the commonly used wavelet denoising algorithm based on wavelet packet decomposition of the signal and the noise reduction algorithm, and the decomposition denoising wavelet threshold denoising method and the wavelet packet decomposition denoising algorithm is applied to diesel engine surface vibration signal. Examples prove that: compared with the low pass filtering denoising technique based on the traditional $\mathrm{Fu}$ Liye analysis, wavelet analysis the incomparable superiority display in non-stationary signal denoising.

This paper has the following problem: denoising algorithm in various summary, the parameter selection problem exists for various algorithms, only limited to qualitative analysis, calculation cannot give general rules and quantitative, can only be determined in the experiments according to the specific situation.

\section{References}

[1]Tang Bao - ping, Yang Changqi, Tan Shanwen et al. The translation invariant wavelet denoising method and its application to [J]. Journal of Chongqing University (NATURAL SCIENCE) based on, 2002, 25 (3):1-5.

[2] Zhang Weiqiang, Mr. Xu early morning. A translation invariant wavelet threshold denoising algorithm based on [J]. modern electronic technique, 2003, (6):29-31.

[3] Wang Bin, Tian Jin Wen, Peng Fuyuan. Wavelet to process the maximum pair mode noise in [J]. Journal of Huazhong Polytechnic University, 1997, 25 (11):61-63.

[4] Zhang Liang, Du Haiping, history Xizhi. Wavelet analysis of vibration signals of diesel engine noise reduction based on [J]. data acquisition and processing, 2000, 15 (4):525-527.

[5]Huang Qiang, Liu Yongchang. High Shilun, wavelet denoising vibration diagnosis in diesel engine in the application of a [J]. small internal combustion engine and motorcycle, 2003, 32 (5):7-10. 